\title{
Motivationale Faktoren für die Präferenz der Fachrichtung Allgemeinmedizin - eine quantitative Untersuchung unter Medizinstudierenden NRWs für eine zielgerichtete Nachwuchsgewinnung
}

\author{
Motivation for Specialisation in General Practice - A Quantitative \\ Study of NRW's Medical Students in Support of Targeted Recruitment
}

\author{
Autoren \\ Institute \\ 1 Institut für Allgemeinmedizin und Familienmedizin, \\ Universität Witten/Herdecke, Witten \\ 2 Lehrstuhl für Forschungsmethodik und Statistik in der \\ Psychologie, Universität Witten/Herdecke, Witten \\ 3 Abteilung für Allgemein- und Familienmedizin am \\ Zentrum für Public Health, Medizinische Universität Wien \\ 4 Service épidémiologie et statistiques, Direction de la santé, \\ Luxembourg
}

Greta Joan Funkhouser ${ }^{1}$, Thomas Ostermann², Andreas Sönnichsen¹, 3, Katharina K. Pucher¹, 4

\section{Schlüsselwörter}

Motivationsfaktoren, Allgemeinmedizin, Fachrichtungswahl, gezielte Nachwuchsgewinnung, Medizinstudierende,

Fachrichtung

Key words

Motivational factors, career choice, targeted recruitment, medical students, speciality, general practice

\author{
Bibliografie \\ DOI https://doi.org/10.1055/a-0777-1782 \\ Online-Publikation: 5.12.2018 \\ Gesundheitswesen 2020; 82: 520-526 \\ (c) Georg Thieme Verlag KG Stuttgart - New York \\ ISSN 0941-3790
}

Korrespondenzadresse

Greta Joan Funkhouser

Institut für Allgemeinmedizin und Familienmedizin

Universität Witten/Herdecke

Alfred-Herrhausen-Straße 50

58455 Witten

greta.funkhouser@gmail.com

\section{ZUSAMMENFASSUNG}

Ziel derStudie In Anbetracht des prognostizierten Allgemeinmediziner-Mangels führten wir eine Online-Umfrage unter allen Medizinstudierenden Nordrhein-Westfalens durch, um Faktoren für die Entscheidung Medizinstudierender zur Fachrichtung Allgemeinmedizin (AM) zu identifizieren und die Stärke der Zusammenhänge zwischen Faktoren und Fachrichtungswahl zu quantifizieren.
Methodik Um Beweggründe für und gegen die Fachrichtungswahl AM zu identifizieren wurde eine Faktorenanalyse mit den Angaben von 3976 Umfrageteilnehmern (22,6\% aller eingeladenen Medizinstudierenden) durchgeführt. Die Stärke der Zusammenhänge zwischen extrahierten Faktoren und Fachrichtungswahl wurden mittels Regressionsanalysen für 2862 Teilnehmer ermittelt, die sich eher für oder gegen AM positioniert hatten. Adjustiert wurde für relevante Populationsmerkmale.

Ergebnisse Fünf Faktoren wurden identifiziert. Signifikante Zusammenhänge zur Fachrichtungswahl AM zeigten 4 dieser Faktoren. „Umfassender Patientenkontakt“ (OR 4,02; 95 \% KI $[3,5-4,6])$, und „flexible Arbeitszeiten“ (OR 1,35; 95 \% KI [1,2$1,5])$ stellen Beweggründe für die Fachrichtungswahl AM dar, obwohl „höhere(s) Karrierechancen, Einkommensmöglichkeiten \& Ansehen in anderen Fachrichtungen“ (OR 1,14; 95 \% KI $[1,0-1,3])$ gesehen werden. Bessere Möglichkeiten der „Spezialisierung, Forschung und Teamarbeit in anderen Fachrichtungen" stehen einer Wahl von AM als Fach entgegen (OR 0,55; $95 \%$ KI $[0,5-0,6])$.

Schlussfolgerungen Der Nachwuchsgewinnung dienlich wären die strukturierte Förderung studentischen Interesses am Patientenkontakt in Lehrpraxen, mehr Spezialisierung, Forschung und Teamarbeit durch verbesserte allgemeinmedizinische Strukturen an Universitäten und eine weitere Flexibilisierung der Arbeitszeiten.

\begin{abstract}
Aim of study Due to forecasted shortage of general practitioners, we conducted an online survey of all medical students in North-Rhine-Westphalia to identify and quantify determinants in medical students' decision to specialize in general practice (GP).

Methodology To identify the motives driving the choice of GP as a career, responses of 3976 participants (22.6\% of medical students invited) were subjected to factor analysis. Relationships between extracted factors and career choice were ascertained via regression analysis adjusted for population characteristics. All participants who had positioned themselves distinctly for/ against GP were included in regression analysis $(n=2862)$.

Results Four of five identified factors were significantly associated with the intended choice of GP. "Comprehensive patient
\end{abstract}


contact" (OR 4.02; $95 \% \mathrm{Cl}$ [3.5-4.6]), and "flexible working hours" (OR 1.35; 95\% Cl [1.2-1.5]) represent factors associated with choosing GP despite the perception of "superior career opportunities, income and status in other specializations" (OR 1.14; $95 \% \mathrm{Cl}$ [1.0-1.3]). Meanwhile, preference for "specialization, research and teamwork in other disciplines" (OR 0.55; $95 \% \mathrm{Cl}[0.5-0.6])$ obstructs the intended choice of GP.
Conclusion GP recruitment can profit from focused sponsorship of students' interest in patient contact within GP clerkships, appropriate GP teaching structure at the universities, enhanced research and teamwork opportunities, and increased flexibility of working hours.

\section{Einleitung}

Der demografische Wandel, die anlaufende Ruhestandswelle, eine wachsende Inanspruchnahme von Teilzeitstellen im ambulanten Bereich, aber auch die noch immer zu geringen Nachwuchszahlen stellen die ambulante Primärversorgung vor große Herausforderungen [1-3]. Sofern es nicht gelingt, mehr Ärzte für diesen Sektor zu gewinnen, droht laut Prognosen der Kassenärztlichen Bundesvereinigung (KBV) bis 2030 eine großflächige Unterversorgung der Patienten, v. a. in ländlichen Gebieten [4]. Daher ist die nachhaltige Gewinnung von Nachwuchs in der Allgemeinmedizin (AM) ein aktuelles Thema für Entscheidungsträger im Gesundheitswesen. Entsprechende Studien zur Fachrichtungswahl von Medizinstudierenden und den Beweggründen hierfür können erste Anhaltspunkte für den Entwicklungsbedarf und zielgerechte Veränderungen liefern. Seit 2011 wurden hierzu drei bundesweite [5-7] und eine regionale [8] Studie veröffentlicht, denen zufolge nur ca. $9 \%$ der Studierenden eine Weiterbildung in der AM $[5,7]$ planen, und auch nur ca. $30 \%$ die AM als eine Option neben anderen Fachrichtungen sehen [6]. Als Beweggründe für oder gegen die Fachrichtungswahl Allgemeinmedizin werden in diesen Studien folgende Faktoren identifiziert: Work-Life-Balance, Familie und Freizeit, Berufsprestige und Einkommen, Teamorientierung, Abwechslung im Beruf, Wissenschaftsorientierung, Patientenorientierung, Karriere, Image, persönliche Ambition, Zukunftsperspektiven, Bürokratische Hemmnisse, hohe Arbeitsbelastung [5-7].

Die statistischen Zusammenhänge zwischen der Fachrichtungswahl Allgemeinmedizin und möglichen Beweggründen wurden bisher nur in 2 regionalen Studien an kleineren Kohorten $(N<1000)$ untersucht $[9,10]$, wobei der Fokus jeweils auf ausgewählten Faktoren lag. Es zeigte sich zum einen, dass das weibliche Geschlecht und das Vorhandensein eines Lehrstuhls für Allgemeinmedizin am Ausbildungsort [9] und zum anderen praktische Erfahrung während des Studiums [10] in starkem Zusammenhang mit der Motivation zu einer hausärztlichen Tätigkeit stehen. Zu ähnlichen Ergebnissen kam eine amerikanische Meta-Analyse [11]. Zusätzlich wiesen bei einem Sample von 659 Studierenden das Alter, die Familie oder Freunde in der AM, das Erwägen des Hausarztberufs zu Studienbeginn, die Bevorzugung eines Arbeitsplatzes in ländlicher Umgebung, ein breites Patientenspektrum und die langzeit-ArztPatienten-Beziehung einen Zusammenhang mit der Fachrichtungswahl AM auf [10]. Sowohl die mit der Fachrichtungswahl Allgemeinmedizin zusammenhängenden Faktoren als auch die Stärke solcher Zusammenhänge können richtungsweisend für das Priorisieren von Entwicklungsbedarfen und zielgerechten Veränderungen sein. Die Ermittlung solcher Zusammenhänge mit den Beweggründen in einer repräsentativen Kohorte ist deshalb essenziell. Mit ca. 19\% stellt Nordrhein-Westfalen (NRW) einen bedeutenden Anteil der insgesamt 92011 zum Wintersemester 2016/2017 in Deutschland eingeschriebenen Nachwuchsmediziner [12] und bietet damit gute Voraussetzungen für eine Studie zu Zusammenhängen zwischen Einstellungen und Charakteristika von Studierenden und der Fachrichtungswahl. Das Ziel der vorliegenden Studie ist es, anhand eines repräsentativen Samples aus allen Medizinstudierenden NRWs Faktoren für die Entscheidung Medizinstudierender zur Wahl der Fachrichtung Allgemeinmedizin zu extrahieren und deren Zusammenhang mit dieser Fachrichtungswahl zu quantifizieren, um daraus Empfehlungen zur Nachwuchsgewinnung abzuleiten.

\section{Methodik}

Als Grundlage für diese Studie dienen die Daten einer Online-Umfrage. Über den Kompetenzverbund Allgemeinmedizin NRW (http://www.allgemeinmedizin-nrw.de), einen Zusammenschluss der Abteilungen für Allgemeinmedizin der acht medizinischen Fakultäten NRWs (Aachen, Bonn, Bochum, Duisburg-Essen, Düsseldorf, Köln, Münster, Witten/Herdecke) wird eine flächendeckende Beforschung des medizinischen Nachwuchses in NRW ermöglicht.

Im Rahmen der vorliegenden Querschnittstudie wurden im Zeitraum vom 31.10.2016 bis zum 31.01.2017 (WS 2016/2017) alle in NRW immatrikulierten Medizinstudierenden zur Teilnahme an einer Online-Umfrage zu ihrer Fachrichtungswahl und ihren Beweggründen für ihre Wahl eingeladen. Abhängig vom Studienstandort und dessen Vorgaben wurde über E-Mail-Verteiler der Studiendekanante, Ausschreibungen (Schwarzes Brett), soziale Medien und Dozenten rekrutiert. Während des Erhebungszeitraumes fanden nach Möglichkeit bis zu 2 Nachfassaktionen pro Standort im November und Januar statt, um eine möglichst hohe Rücklaufquote zu erzielen. Als Anreiz zur Teilnahme wurden Bargeld und Thieme-Gutscheine (ohne sonstige Beteiligung des Thieme-Verlags an dieser Studie gespendet) unter den Teilnehmenden verlost.

\section{Online-Umfrage}

Der Fragenkatalog für die Online-Umfrage ist in Zusammenarbeit mit dem Institut für Allgemeinmedizin und evidenzbasierte Versorgungsforschung der Medizinischen Universität Graz entwickelt worden, da die Umfrage auch in Österreich durchgeführt werden sollte. Die Fragen wurden teils neu konzipiert und teils aus bestehenden Fragebögen übernommen [5, 7]. Der finale Fragenkatalog enthielt 34 überwiegend geschlossene Fragen. Gegenstand der gegenwärtigen Untersuchung waren Fragen zu den demographischen Daten der Teilnehmenden (z. B. Alter, Geschlecht, Studienabschnitt, Studienstandort, Anzahl der Kinder, Herkunftsort, Verwandte in der AM), 
Fragen zur Erfahrung mit der praktischen und theoretischen allgemeinmedizinischen Lehre (z. B. „Haben Sie bereits theoretische Erfahrung (z. B. Vorlesungen, Seminare) mit der Allgemeinmedizin?“, „Haben Sie bereits praktische Erfahrung mit der Allgemeinmedizin?“), sowie 5 Fragenblöcke, die 24 mögliche Beweggründe für und gegen die Fachrichtungswahl Allgemeinmedizin enthielten (z. B. „Der Hausarztberuf ist für mich attraktiv, weil ...., „Eine Facharztqualifikation in einem anderen Fach ist für mich attraktiver, weil ...“). Die Studierenden konnten hier in Form einer 5-Punkte-Likert-Skala von „trifft zu“ bis „trifft nicht zu“ angeben, was für sie den Hausarztberuf attraktiv macht (z. B. der umfangreiche und unterschiedliche Patientenkontakt) oder weshalb eine andere Fachrichtung der Allgemeinmedizin vorzuziehen sein könnte (z. B. weil man dort sein Wissen speziell vertiefen kann). Zusätzlich wurde die angestrebte Fachrichtungswahl der Teilnehmenden erfragt und als Pflichtfrage programmiert („Welchen Ausbildungsweg möchten Sie aus heutiger Sicht einschlagen?“). Es konnte mit einer von 4 Antwortoptionen auf die Frage geantwortet werden: „für mich kommt nur Allgemeinmedizin in Frage“, „Allgemeinmedizin ist neben anderen Fachrichtungen eine Option“, „für mich kommt nur ein anderer Facharzt in Frage“ und „ich weiß es noch nicht“.

Im September 2016 wurde der Fragenkatalog in Einzelinterviews mit je zehn Studierenden der Universität Witten/Herdecke und der Universität Graz auf Vollständigkeit und Verständlichkeit überprüft und entsprechend angepasst (comprehension probing) [13].

In der Online-Umfrage wurde zugunsten der Anonymität der Teilnehmenden ausschließlich mit Cookies gearbeitet, um die Wahrscheinlichkeit einer wiederholten Teilnahme gering zu halten. Darüber hinaus wurden einige Fragen als Pflichtfragen programmiert, um zu verhindern, dass Teilnehmer sich ohne Angaben durch die Umfrage klicken. Für die Datenerhebung wurde Limesurvey verwendet (https://www.limesurvey.org/de/).

\section{Statistische Auswertung}

Eine Faktorenanalyse mit Hauptkomponentenanalyse und Varimax Rotation wurde zur Dimensionsreduktion der Items ( $N=24)$ zu den Beweggründen für die Fachrichtungswahl durchgeführt. Die Anzahl der Faktoren wurde nach dem Kaiser-Guttman-Kriterium bestimmt. Die Eignung der Daten für die Faktorenanalyse wurde nach dem Kaiser-Meyer-Olkin-Kriterium geprüft. Bei inhaltlichen Überlappungen, wurde das Item mit der geringsten Anzahl fehlender Werte eingeschlossen. Während der Analyse wurden zusätzlich Items exkludiert, die eine Faktor-Ladung < 0,5 aufwiesen, auf mehr als einen Faktor luden, oder laut Einschätzung der Arbeitsgruppe nach ausführlicher Diskussion von vernachlässigbarer Bedeutung für diese Fragestellung waren (z. B. „... weil man mit einer Kassenstelle nicht genug Zeit für den/die einzelne/n Patienten/in und Gespräche hat). Die zusammenhängenden Items wurden anschließend jeweils unter einer gemeinsamen Skala zusammengefasst.

Fehlende Werte der Skalen wurden mithilfe des EM-Algorithmus ersetzt, um eine möglichst hohe Fallzahl für die statistischen Analysen gewährleisten zu können [14]. Zusätzlich wurde eine Complete-Case-Analysis (CCA) durchgeführt, bei der fehlende Werte exkludiert wurden. Zur Bestimmung der internen Konsistenz wurde Cronbach's Alpha für die unterschiedlichen Skalen berechnet und nach Bland und Altman (1997) interpretiert.
Im Hinblick auf die Fragestellung der vorliegenden Studie wurden Fälle, in denen die Pflichtfrage zur Fachrichtungswahl nicht oder mit „weiß ich nicht“ beantwortet war, exkludiert. Zudem wurden Fälle mit der Fachrichtungswahl „Für mich kommt nur AM in Frage“ und „Allgemeinmedizin ist neben anderen Fachrichtungen eine Option“ zur Gruppe „AM-Interessierte“ zusammengefasst. In die weiteren Analysen wurden nur AM-Interessierte und Nicht-AM-Interessierte einbezogen.

Für die Populationsmerkmale wurden Häufigkeiten, Mittelwerte und Unterschiede zwischen den Gruppen mittels T-Test und ChiQuadrat-Test ermittelt.

Zusammenhänge zwischen den Beweggründen der Studierenden und ihrer Fachrichtungswahl wurden mit einer logistischen Regression errechnet. Als abhängige Variable wurden die Gruppenvariable zur Fachrichtungswahl (AM-Interessierte vs. Nicht-AM-Interessierte) und als unabhängige Variable die Skalen zu den Beweggründen eingeschlossen. Adjustiert wurde für Populationsmerkmale, die signifikante Unterschiede $(\alpha<0,01)$ zwischen den Gruppen zeigten (Geschlecht, Kinder, Verwandte in der AM). Die logistische Regression wurde zur Sensitivitätsanalyse mit den Complete-Cases-Skalen wiederholt. Odds Ratios und deren Konfidenzintervalle wurden verwendet, um Zusammenhänge zu interpretieren. Die erhobenen Daten wurden statistisch mit SPSS 23 (IBM Statistics) ausgewertet.

\section{Ergebnisse}

\section{Rücklauf}

Laut Studiendekanaten waren im Wintersemester (WS) 2016/2017 insgesamt 17554 Medizinstudierende in NRW immatrikuliert. Davon haben 3976 (22,6\%) an der Umfrage teilgenommen und konnten in die Faktorenanalyse eingeschlossen werden. 3414 $(19,4 \%)$ haben die Pflichtfrage zur Fachrichtungswahl beantwortet, davon $N=121$ (3,5\%) „für mich kommt nur Allgemeinmedizin in Frage“; $N=1584$ (46,4\%) ,,Allgemeinmedizin ist neben anderen Fachrichtungen eine Option“; N = 1157 (33,9\%) „für mich kommt nur ein anderer Facharzt in Frage“; N= 552 (16,17\%) „ich weiß es noch nicht“. Insgesamt N = 1705 Teilnehmer konnten der Gruppe „AM-Interessierte“ zugeordnet werden. Gemeinsam mit den 1157 Nicht-AM-Interessierten (,für mich kommt nur ein anderer Facharzt in Frage“) konnten somit insgesamt 2862 (16,3\%) Medizinstudierende in die Analysen eingeschlossen werden ( $>$ Abb. 1). Die 522 Studierenden, die „ich weiß es noch nicht“ angegeben hatten, wurden exkludiert.

Die Anzahl eingeschlossener Medizinstudierenden variierte unter den Hochschulstandorten zwischen 7,6 und 27,3\%, wobei die Anzahl der zum Wintersemester 2016/2017 (WS 2016/17) eingeschriebenen Medizinstudierenden bei den jeweiligen Studiendekanaten erfragt wurde.

\section{Faktorenanalyse und Reliabilität}

Das Maß der Stichprobeneignung nach dem Kaiser-Meyer-OlkinKriterium lag bei 0,867 , womit sich die Daten nach Möhring \& Schlütz (2013) für die anschließende Faktorenanalyse eigneten [15]. Die Faktorenanalyse der Beweggründe für oder gegen den Hausarztberuf ergab eine Fünf-Faktoren-Lösung, bei der 18 der ursprüng- 


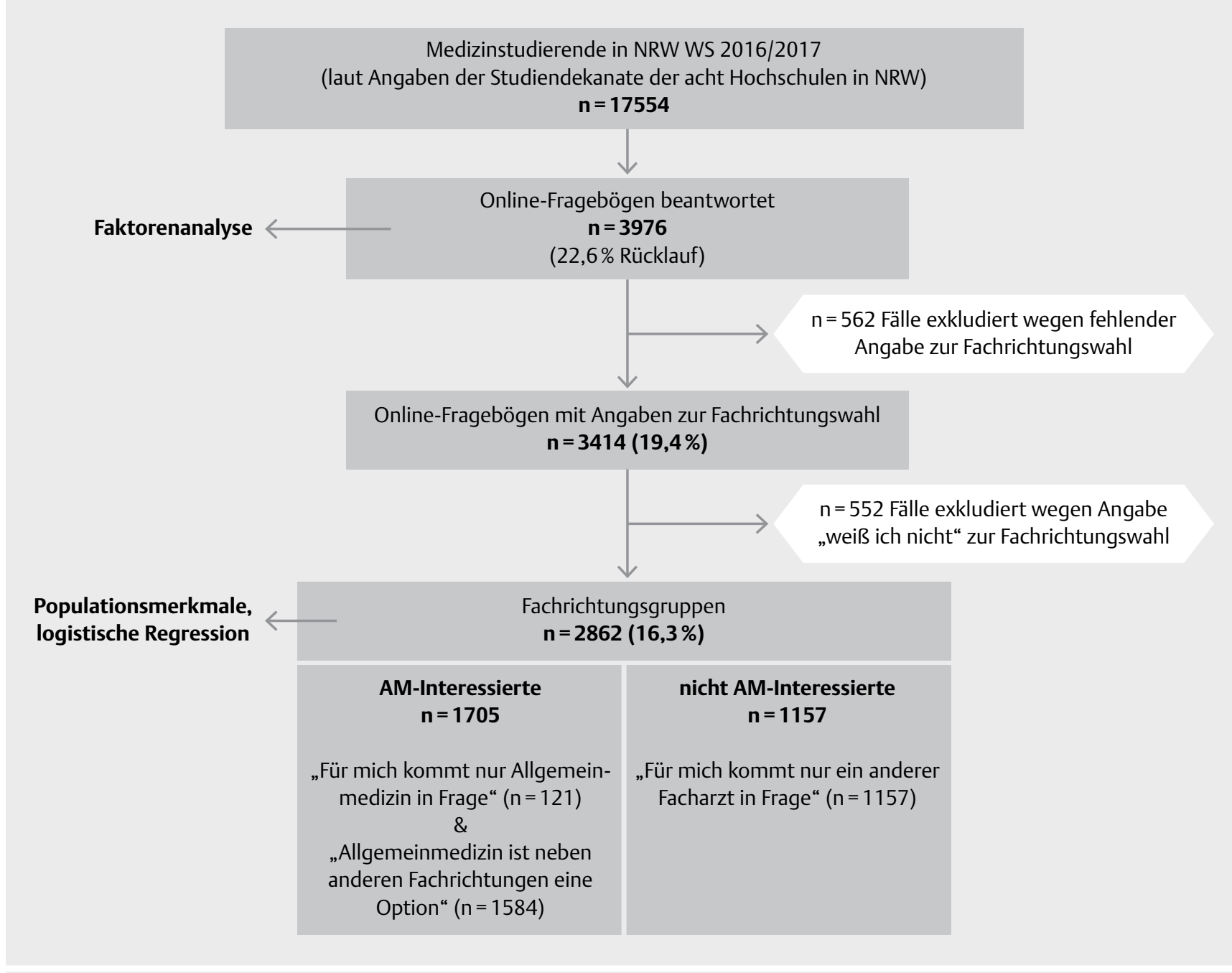

Abb. 1 WS, Wintersemester; AM, Allgemeinmedizin.

lich 24 Items eingeschlossen werden konnten (• Tab. 1). Der erste Faktor „umfassender Patientenkontakt“ erklärte mit sieben Items $26,4 \%$ der Gesamtvarianz und wies mit einem Cronbach's Alpha von $\alpha=0,89$ eine hohe Reliabilität auf. Faktor 2 beschrieb „höhere(s) Karrierechancen, Einkommen \& Ansehen in anderen Fachrichtungen" und setzte sich aus 3 Items zusammen (13,4\% erklärte Varianz, $\alpha=0,80$ ). Faktor 3 ,flexible Arbeitsbedingungen“ wurde aus 2 Items gebildet, erklärte 9,5\% der Gesamtvarianz und wies mit $\alpha=0,79$ ebenfalls eine gute Reliabilität auf. „Nachteile der Niederlassung“ (3 Items, 7,1 \% der Gesamtvarianz, $\alpha=0,55$ ) und „mehr Spezialisierung, Forschung und Teamarbeit in anderen Fachrichtungen“ (3 Items, 5,9\% der Gesamtvarianz, $\alpha=0,50$ ) bildeten die Faktoren 4 und 5, die im Gegensatz zu den ersten 3 Faktoren Cornbach's Alpha Werte $<0,70$ ergaben und damit schwächere Reliabilität aufweisen. Die erklärte Gesamtvarianz der 5 Faktoren lag bei 62,3\%. Die korrigierte Inter-Skala-Korrelation zeigte eine hohe interne Konsistenz; Sie lag für alle Items im Referenzbereich 0,2-0,8.

\section{Unterschiede in Populationsmerkmalen (AM- Interessierte und Nicht-AM-Interessierte)}

AM-Interessierte sind häufiger weiblich, haben häufiger Kinder und haben öfter Eltern oder nahe Verwandte, die in der AM tätig sind ( $\triangleright$ Tab. 2).

\section{Stärke der Zusammenhänge zwischen Fachrichtungswahl und Beweggründen}

- Tab. 3 zeigt einen statistisch signifikanten Zusammenhang zwischen der Fachrichtungswahl und den Faktoren 1, 2, 3 und 5. Studierende mit Wunsch nach umfassendem Patientenkontakt ziehen mit einer fast 4-mal höheren Chance die AM in Betracht (OR 4,02; $95 \% \mathrm{KI}[3,5 ; 4,6])$. Ebenfalls steigt die Wahrscheinlichkeit in die AM zu gehen, wenn die Arbeitszeiten als flexibel und gut mit der Familie vereinbar gesehen werden, (OR 1,35; 95 \% KI [1,2; 1,5]). Darüber hinaus wählen Studierende die Fachrichtung AM, obwohl sie der Meinung sind, dass Karrierechancen, Einkommen und Ansehen in anderen Fachrichtungen höher sind (OR 1, 14; $95 \%$ KI [1,0; 1,3]) und gehen seltener in die AM, wenn sie bessere Möglichkeiten für Spezialisierung, Forschung und Teamarbeit in anderen Fachrich- 
- Tab. 1 Faktorenanalyse und interne Konsistenz ( $\mathrm{N}=3976)$.

\begin{tabular}{|c|c|c|c|c|}
\hline Item & $\begin{array}{l}\text { Erklärte } \\
\text { Varianz }\end{array}$ & $\begin{array}{l}\text { Faktor } \\
\text { Ladung }\end{array}$ & $\begin{array}{l}\text { Korrigierte Inter- } \\
\text { Skala-Korrelation }\end{array}$ & $\begin{array}{l}\text { Item Mittel- } \\
\text { wert }(S D)\end{array}$ \\
\hline Faktor 1: umfassender Patientenkontakt $(\alpha=0,89)$ & $26,4 \%$ & & & \\
\hline \multicolumn{5}{|l|}{ Der Hausarztberuf ist für mich attraktiv, ... } \\
\hline $\begin{array}{l}\text { weil ich als Allgemeinmediziner/in oft ganze Familien über Generationen hinweg ärztlich } \\
\text { begleite }\end{array}$ & & 0,816 & 0,743 & $3,70(1,26)$ \\
\hline weil ich für meine Patienten/innen der erste Ansprechpartner bin & & 0,807 & 0,722 & $3,77(1,15)$ \\
\hline wegen der persönlichen und langjährigen Beziehungen zu den Patienten/innen & & 0,796 & 0,738 & $3,94(1,20)$ \\
\hline $\begin{array}{l}\text { weil ich bei der Versorgung meiner Patienten/innen neben den körperlichen Beschwerden } \\
\text { auch psychologische, soziale und kulturelle Einflussfaktoren berücksichtigen kann }\end{array}$ & & 0,778 & 0,697 & $3,72(1,25)$ \\
\hline weil ich sowohl banale Krankheiten wie auch Notfälle selber versorgen kann und muss & & 0,755 & 0,671 & $3,69(1,18)$ \\
\hline weil Patienten/innen mit chronischen Krankheiten behandelt werden & & 0,732 & 0,619 & $3,07(1,23$ \\
\hline wegen der vielen unterschiedlichen Patientenkontakte & & 0,717 & 0,653 & $3,89(1,20)$ \\
\hline $\begin{array}{l}\text { Faktor 2: höhere(s) Karrierechancen, Einkommen \& Ansehen in anderen Fachrich- } \\
\text { tungen }(\alpha=0,80)\end{array}$ & $13,4 \%$ & & & \\
\hline \multicolumn{5}{|l|}{ Eine Facharztqualifikation in einem anderen Fach ist für mich attraktiver, ... } \\
\hline weil man dort mehr verdienen kann & & 0,855 & 0,634 & $2,79(1,38)$ \\
\hline weil ich dort bessere Karrierechancen habe & & 0,812 & 0,674 & $2,98(1,39)$ \\
\hline weil man dort ein besseres Ansehen erlangen kann & & 0,778 & 0,605 & $2,46(1,33)$ \\
\hline Faktor 3: flexible Arbeitszeiten $(\alpha=0,79)$ & $9,5 \%$ & & & \\
\hline \multicolumn{5}{|l|}{ Der Hausarztberuf ist für mich attraktiv, ... } \\
\hline wegen der guten Arbeitszeiten und der flexiblen Zeiteinteilung & & 0,897 & 0,658 & $3,59(1,31)$ \\
\hline wegen der Vereinbarkeit von Beruf und Familie & & 0,875 & 0,658 & $3,62(1,21)$ \\
\hline Faktor 4: Nachteile der Niederlassung $(\alpha=0,55)$ & $7,1 \%$ & & & \\
\hline \multicolumn{5}{|l|}{ Aus heutiger Sicht kann mich davon abhalten Allgemeinmediziner/in zu werden, ... } \\
\hline dass ich zu viele administrative Tätigkeiten hätte & & 0,745 & 0,398 & $3,34(1,23)$ \\
\hline dass mir das wirtschaftliche Risiko zu groß ist & & 0,706 & 0,369 & $3,09(1,30)$ \\
\hline dass ich von den Krankenkassen zu viele Vorgaben gemacht bekäme & & 0,646 & 0,322 & $3,95(1,04)$ \\
\hline $\begin{array}{l}\text { Faktor 5: mehr Spezialisierung, Forschung und Teamarbeit in anderen Fachrichtun- } \\
\text { gen }(\alpha=0,50)\end{array}$ & $5,9 \%$ & & & \\
\hline \multicolumn{5}{|l|}{ Eine Facharztqualifikation in einem anderen Fach ist für mich attraktiver, ... } \\
\hline weil man dort sein Wissen speziell vertiefen kann & & 0,721 & 0,330 & $3,93(1,16)$ \\
\hline weil ich als Facharzt/ärztin besser in der Forschung arbeiten kann & & 0,658 & 0,327 & $2,64(1,49)$ \\
\hline weil Teamarbeit als Facharzt/ärztin leichter ist] & & 0,637 & 0,284 & $2,85(1,29)$ \\
\hline
\end{tabular}

tungen sehen (OR 0,55; $95 \%$ KI [0,5; 0,6]). Die Ergebnisse der Complete-Case-Analysis unterschieden sich nur unwesentlich von der Analyse mit imputierten Daten für fehlende Werte.

\section{Diskussion}

Ziel der Studie war es, den statistischen Zusammenhang zwischen möglichen Beweggründen von Medizinstudierenden und der Fachrichtungswahl Allgemeinmedizin darzustellen. Damit schließt diese Studie eine vorhandene Lücke in der bisherigen Forschungslandschaft und ermöglicht eine Priorisierung von Handlungsmaßnahmen, um den allgemeinmedizinischen Nachwuchs zu stärken.

Als wichtigstes Ergebnis ist festzustellen, dass der Wunsch nach umfassendem Patientenkontakt (Faktor 1) mit der höchsten OR und damit der stärksten Äußerung des Berufswunsches Hausarzt assoziiert ist. Das deckt sich mit den Ergebnissen einer lokalen Studie aus Sachsen an einer kleineren Kohorte $(N=659)$, die ebenfalls einen positiven Zusammenhang zwischen dem Wunsch, Allgemeinmediziner zu werden, und der Langzeit-Arzt-Patienten-Beziehung, sowie dem breiten Patientenspektrum zeigte [10]. Es ist bekannt, dass Patientenkontakt in der Lehrpraxis bestehendes Interesse an der Primär-Versorgung verstärken kann [16] und fortwährende praktische Erfahrung während der Ausbildung mit einem höheren Anteil an AM-wählenden Studierenden assoziiert ist [11]. Dementsprechend sollten die praktische Erfahrung mit Patienten und das studentische Interesse am Patientenkontakt mehr in den Vordergrund der studentischen Ausbildung rücken. Um mehr Medizinstudierende für den Beruf des Hausarztes zu gewinnen, kann die praktische Ausbildung in den Lehrpraxen besser strukturiert werden, um bestehende AM-Interessenten zu halten. Um flächendeckend eine hohe Qualität für studentische Erfahrungen in den Lehrpraxen zu erreichen, ist zentralisierte Qualitätssicherung notwendig. Die Vereinigung der Hochschullehrer und Lehrbeauftragten für Allgemeinmedizin legte in diesem Sinne bereits Strukturempfehlungen für die praktische Ausbildung in Lehrpraxen vor [17]. Solche Ansätze sollten gefördert und in Zusammenarbeit mit den Lehrärzten ausgebaut werden. Zentralisierter Austausch über Bedürfnisse der Lehrpraxen, Universitäten und Studierenden ist hierbei wünschenswert. 
\ Tab. 2 Populationsbeschreibung nach Fachrichtungsgruppen „AM-Interessierte“ \& „Nicht-AM-Interessierte“ mit Darstellung der Unterschiede zwischen den Gruppen.

\begin{tabular}{|c|c|c|c|c|}
\hline \multirow[t]{3}{*}{ Populationsmerkmale } & \multirow[t]{2}{*}{ fehlende Werte } & \multicolumn{2}{|c|}{ Fachrichtungswahl } & \multirow{2}{*}{$\begin{array}{l}\text { Unterschiede } \\
\text { p-Wert }\end{array}$} \\
\hline & & AM-Interessierte & Nicht-AM-Interessierte & \\
\hline & $\mathrm{N}(\%)$ & $N=1705$ & $N=1157$ & \\
\hline Alter & $35(1,2 \%)$ & $24,3(S D 4,8)$ & $24,1(S D 4,2)$ & 0,161 \\
\hline Geschlecht (weiblich) & & $1220(71,6 \%)$ & $742(64,1 \%)$ & $0,000^{*}$ \\
\hline Studienabschnitt & & & & 0,075 \\
\hline Vorklinischer Abschnitt & & $606(35,5 \%)$ & $365(31,5 \%)$ & \\
\hline Klinischer Abschnitt & & $904(53,0 \%)$ & $644(55,7 \%)$ & \\
\hline Praktisches Jahr & & $195(11,4 \%)$ & $148(12,8 \%)$ & \\
\hline Praktische Erfahrungen mit der AM & $25(0,9 \%)$ & $1123(66,5 \%)$ & $751(65,4 \%)$ & 0,519 \\
\hline Theoretische Erfahrungen mit der AM & $81(2,8 \%)$ & $1247(75,3 \%)$ & $860(76,4 \%)$ & 0,534 \\
\hline Studiengang & & & & 0,310 \\
\hline Modellstudien-/Reformstudiengang & & $1075(63,0 \%)$ & $751(64,9 \%)$ & \\
\hline Regelstudiengang & & $630(37,0 \%)$ & $406(35,1 \%)$ & \\
\hline Medizinstudierende mit Kindern & $406(14,2 \%)$ & $89(6,0 \%)$ & $29(3,0 \%)$ & $0,000^{*}$ \\
\hline $\begin{array}{l}\text { Medizinstudierende mit Eltern oder anderen nahen } \\
\text { Verwandten, die hausärztlich tätig sind/waren }\end{array}$ & $411(14,4 \%)$ & $240(16,3 \%)$ & $93(9,5 \%)$ & $0,000^{*}$ \\
\hline
\end{tabular}

> Tab. 3 Ergebnisse der Logistischen Regression (Analyse mit imputierten Daten für fehlende Werte \& Complete Cases) mit Fachrichtungswahl („AM-Interessierte“ vs. „Nicht-AM-Interessierte“) als abhängige Variable.

\begin{tabular}{|c|c|c|c|c|c|c|}
\hline \multirow[t]{2}{*}{ Items } & \multicolumn{3}{|c|}{$\begin{array}{l}\text { Analyse mit imputierten Daten für } \\
\text { fehlende Werte }\end{array}$} & \multicolumn{3}{|c|}{ Complete Cases (CC) } \\
\hline & $p$ & OR & $\mathrm{Cl}$ & $p$ & OR & $\mathrm{Cl}$ \\
\hline Faktor 1: umfassender Patientenkontakt & 0,000 & 4,02 & $3,5-4,6$ & 0,000 & 3,77 & $3,2-4,4$ \\
\hline $\begin{array}{l}\text { Faktor 2: höhere(s) Karrierechancen Einkommen \& Ansehen in } \\
\text { anderen Fachrichtungen }\end{array}$ & 0,007 & 1,14 & $1,0-1,3$ & 0,010 & 1,16 & $1,0-1,3$ \\
\hline Faktor 3: flexible Arbeitszeiten & 0,000 & 1,35 & $1,2-1,5$ & 0,000 & 1,39 & $1,2-1,5$ \\
\hline Faktor 4: Nachteile der Niederlassung & 0,190 & 1,10 & $1,0-1,3$ & 0,181 & 1,10 & $1,0-1,3$ \\
\hline $\begin{array}{l}\text { Faktor 5: mehr Spezialisierung, Forschung und Teamarbeit in } \\
\text { anderen Fachrichtungen }\end{array}$ & 0,000 & 0,55 & $0,5-0,6$ & 0,000 & 0,54 & $0,5-0,6$ \\
\hline Geschlecht & 0,157 & 0,86 & $0,7-1,1$ & 0,491 & 0,92 & $0,7-1,2$ \\
\hline Kinder & 0,003 & 0,44 & $0,2-0,8$ & 0,085 & 0,59 & $0,3-1,1$ \\
\hline Eltern bzw. Verwandte in der AM & 0,050 & 0,73 & $0,5-1,0$ & 0,163 & 0,78 & $0,5-1,1$ \\
\hline
\end{tabular}

Zweitrangig stand der Faktor „Teamarbeit, Forschung und Spezialisierung in anderen Fachrichtungen " mit der Berufswahl AM in signifikantem Zusammenhang. Studierende, denen diese Merkmale wichtig sind, streben eher keine allgemeinmedizinische Laufbahn an. Hier sind nun die Universitäten gefragt: Bereits an über 70\% der deutschen Fakultäten sind selbstständige Institute oder Abteilungen für AM eingerichtet oder in Planung $[18,19]$. Diese Entwicklung ist unerlässlich für den Forschungsfortschritt, die Wahrnehmung der Allgemeinmedizin als selbstständige Fachrichtung und die Vernetzung innerhalb der Disziplin [19]. Es gilt nun, diesen Weg konsequent weiterzugehen. Der Stellenwert der Allgemeinmedizin als forschendes Fach, das Spezialkenntnisse und Teamarbeit erfordert, muss aufgewertet werden. Dies kann sowohl durch eine Förderung allgemeinmedizinischer Strukturen an den Universitäten als auch durch eine intensivierte, wissenschaftlich fundierte Lehre in der Allgemeinmedizin geschehen.

Hinsichtlich der Signifikanz steht der Zusammenhang zwischen guter Vereinbarkeit von Beruf und Familie im Hausarztberuf und der Fachrichtungswahl AM an dritter Stelle. Die hausärztliche Tätigkeit wird schon jetzt als flexibel und gut vereinbar mit Freizeit und Familie angesehen. Hier könnten noch weitere Gestaltungsspielräume im niedergelassenen Bereich (Job-Sharing, Anstellung, Teilzulassung usw.) mehr Nachwuchs in die Primärversorgung locken. Ergänzend fällt der hohe Frauenanteil der AM-Interessierten sowohl in der vorliegenden, als auch in anderen Studien auf [6, 7], was teilweise dem steigenden Anteil weiblicher Medizinstudierender zuzuschreiben ist [20]. Für Frauen hat die Vereinbarkeit von Familie und Beruf häufig eine hohe Relevanz [7].

Zuletzt zeigte sich, dass AM-Interessierte zwar erkennen, dass Karrierechancen, Einkommen und Ansehen in anderen Fachrichtungen höher sind (Faktor 2) [6-8], aber dennoch gewillt sind, AM zu wählen. Zukünftig sollten Hausärzte hinsichtlich Karrierechancen, Einkommen und Ansehen den anderen Fächern gleichgestellt werden, um die Attraktivität des Berufs auch für jene Studierende zu steigern, für die diese Berufsaspekte eine höhere Priorität darstellen. 


\section{Limitationen \& Stärken}

Soweit vergleichbare Fragestellungen untersucht wurden, decken sich die beschriebenen Ergebnisse unserer Studie mit denen vorangegangener Studien [6-8]. Hinsichtlich Geschlecht, Alter und Nationalität ist die Kohorte der vorliegenden Studie [69,3\% Frauenanteil, 23,0 Jahre (Median), 82,3\% deutsche Studierende] mit der Gesamtheit der Medizinstudierenden in NRW vergleichbar [62,4\% Frauenanteil, 25,0 Jahre (Median), 87,4\% deutsche Studierende] [21, 22] was für eine gute Repräsentativität unseres Kollektivs hinsichtlich der mit der Wahl der Fachrichtung AM zusammenhängender Beweggründe spricht. Dies ist nicht zuletzt der Teilnahme aller Hochschulstandorte in NRW, der hohen Teilnehmerzahl, und der Verteilung des Kollektivs auf mehrere Hochschulstandorte und alle Studienabschnitte geschuldet. Rückschlüsse auf andere Bundesländer sind nur begrenzt möglich angesichts der unterschiedlichen, bundeslandspezifischen Kontexte. Auch fehlt es an vergleichbaren Studien aus anderen Bundesländern für einen direkten Vergleich.

Ein weiterer starker Aspekt der vorliegenden Studie ist, dass direkt nach Beweggründen für die Fachrichtungswahl gefragt wurde, was es ermöglichte motivationale Faktoren zu identifizieren. Trotz der Stärken dieser Studie können der Rücklauf von 22,6\% und die teilweise unvollständig ausgefüllten Fragebögen nicht ignoriert werden. Auch eine Mehrfachteilnahme oder ein Weitergeben des Umfrage-Links an Personen außerhalb der Zielgruppe kann trotz Cookie-Einstellungen, die ein doppeltes Ausfüllen von einem Computer aus blockierten, nicht mit Sicherheit ausgeschlossen werden.

\section{FAZIT}

- Der Wunsch nach umfassendem Patientenkontakt ist am stärksten mit der Fachrichtungswahl AM assoziiert.

- Zentralisierter Austausch über die Bedürfnisse der Lehrpraxen, Universitäten und Studierenden könnte flächendeckend die praktische Erfahrung der Studierenden aufwerten und dadurch AM-Interessierte Studierende halten.

- Der Stellenwert der Allgemeinmedizin als forschendes Fach, das Spezialkenntnisse und Teamarbeit erfordert, muss aufgewertet werden, um auch Studierenden mit solchen Prioritäten entgegenzukommen.

- Gute Vereinbarkeit von Beruf und Familie im Hausarztberuf steht in positivem Zusammenhang mit der Fachrichtungswahl AM. Weitere Gestaltungsspielräume im niedergelassenen Bereich wären der Nachwuchsgewinnung dienlich.

- Hausärzte sollten hinsichtlich Karrierechancen, Einkommen und Ansehen den anderen Fächern gleichgestellt werden, um das Interesse der Studierenden an der AM zu fördern.

Interessenkonflikt

Die Autoren erklären, dass kein Interessenkonflikt besteht.
Literatur

[1] Bundesregierung. Jedes Alter zählt. Die Demografiestrategie der Bundesregierung. Berlin: Bundesministerium des Innern; 2015

[2] Hillienhof A. Ärztestatistik: Arztzahlen wachsen zu langsam für den Versorgungsbedarf. Dtsch Arztebl International 2017; 114: A-886

[3] Bundesärztekammer. Ärztestatistik 2016. Die Schere zwischen Behandlungsbedarf und Behandlungskapazitäten öffnet sich. Berlin: Bundesärztekammer online; 2017

[4] KBV. Deutschlandweite Projektion 2030 - Arztzahlentwicklung in Deutschland. Präsentation: Pressekonferenz 2016

[5] Hartmannbund. Medizinstudium 2020 Plus. In, Medizinstudium 2020 Plus. Berlin: Hartmannbund

[6] Gibis B, Heinz A, Jacob R et al. Berufserwartungen von Medizinstudierenden. Dtsch Arztebl International 2012; 109: 327-332. doi:10.3238/ arztebl.2012.0327

[7] Jacob R, Kopp J, Schultz S. Berufsmonitoring Medizinstudenten 2014 Ergebnisse einer bundesweiten Befragung. Berlin: KBV, Kassenärztliche Bundesvereinigung; 2015

[8] Kiolbassa K, Miksch A, Hermann K et al. Becoming a general practitioner-which factors have most impact on career choice of medical students? BMC Fam Pract 2011; 12: 25. doi:10.1186/14712296-12-25

[9] Schneider A, Karsch-Völk M, Rupp A et al. Predictors of a positive attitude of medical students towards general practice - a survey of three Bavarian medical faculties. GMS Zeitschrift für Medizinische Ausbildung 2013; 30: Doc45

[10] Deutsch T, Lippmann S, Frese T et al. Who wants to become a general practitioner? Student and curriculum factors associated with choosing a GP career - a multivariable analysis with particular consideration of practice-orientated GP courses. Scand J Prim Health Care 2015; 33: 47-53 doi:10.3109/02813432.2015.1020661

[11] Bland C, Meurer L, Maldonado G. Determinants of primary care specialty choice: a non-statistical meta-analysis of the literature. Acad Med 1995; 70; 620-641

[12] Destatis. Bildung und Kultur: Studierende an Hochschulen. In, Veröffentlichungen des Statistischen Bundesamtes. Wiesbaden: Statistisches Bundesamt; 2017

[13] Porst R. Im Vorfeld der Befragung: Planung, Fragebogenentwicklung, Pretesting: ZUMA 1998

[14] Dempster AP, Laird NM, Rubin DB. Maximum likelihood from incomplete data via the EM algorithm. Journal of the royal statistical society Series B (methodological) 1977; 1-38

[15] Möhring W, Schlütz D. Handbuch standardisierte Erhebungsverfahren in der Kommunikationswissenschaft: Springer-Verlag. 2013

[16] Böhme K, Sachs P, Niebling W et al. Macht das Blockpraktikum Allgemeinmedizin Lust auf den Hausarztberuf? ZFA-Zeitschrift für Allgemeinmedizin 2016; 92: 220-225

[17] GHA. Die Empfehlung der Vereinigung der Hochschullehrer und Lehrbeauftragten für Allgemeinmedizin e.V. In, Z Allg Med 2004; 80: 38-39

[18] Schmid R. Allgemeinmedizin - Auf der Überholspur. Der Allgemeinarzt 2014; 36: 73-74

[19] Richter-Kuhlmann E, Rieser S. Allgemeinmedizin: Ein Fach im Aufwind. Dtsch Arztebl International 2015; 112: A-1866

[20] Hibbeler B, Korzilius H. Arztberuf: Die Medizin wird weiblich. Dtsch Arztebl International 2008; 105: A-609

[21] Information und Technik Nordrhein-Westfalen. Sonderauswertung Geschäftsbereich Statistik. In. Düsseldorf: Information und Technik Nordrhein-Westfalen, Geschäftsbereich Statistik; 2018

[22] Destatis. Sonderauswertung vom Statistischen Bundesamt. In: Statistisches Bundesamt; 2018 